\title{
HoloMentor: A Novel Mixed Reality Surgical Anatomy Curriculum for Robot- Assisted Radical Prostatectomy
}

\author{
Zoe-Athena Papalois ${ }^{a} \quad$ Abdullatif Aydın ${ }^{b} \quad$ Azhar Khan $^{c, d} \quad$ Evangelos Mazaris ${ }^{\mathrm{e}}$ \\ Anand Sivaprakash Rathnasamy Muthusamy ${ }^{f}$ Frank J.M.F. Dor ${ }^{f}$ \\ Prokar Dasguptab, c Kamran Ahmed ${ }^{\text {b, d }}$ \\ aSchool of Medical Education, King's College London, London, UK; b MRC Centre for Transplantation, Guy's Hospital, \\ King's College London, London, UK; 'Department of Urology, Guy's and St. Thomas' NHS Foundation Trust, London, \\ UK; 'Department of Urology, King's College Hospital NHS Foundation Trust, London, UK; 'Department of Urology, \\ Imperial College Healthcare NHS Trust, London, UK; 'Department of Surgery and Cancer Renal and Transplant \\ Centre, Imperial College Healthcare NHS Trust, London, UK
}

\section{Keywords}

Virtual reality - Task analysis - Remote-learning - Robotassisted radical prostatectomy $\cdot$ Surgical training

\begin{abstract}
Objectives: The disruption to surgical training and medical education caused by the global COVID-19 pandemic highlighted the need for realistic, reliable, and engaging educational opportunities available outside of the operating theatre and accessible for trainees of all levels. This article presents the design and development of a virtual reality curriculum which simulates the surgical mentorship experience outside of the operating theatre, with a focus on surgical anatomy and surgical decision-making. Method: This was a multi-institutional study between London's King's College and Imperial College. The index procedure selected for the module was robotic radical prostatectomy. For each stage of the surgical procedure, subject-matter experts $(N=3)$ at King's College London, identified (1) the critical surgical-decision making points, (2) critical anatomical landmarks, and (3) tips and techniques for overcoming intraoperative challenges. Content validity was determined by an independent panel of subject-matter experts $(N=8)$ at Imperial
\end{abstract}

karger@karger.com www.karger.com/esr

Karger $\frac{1}{\%}$
C 2021 The Author(s).

Published by S. Karger AG, Basel

This is an Open Access article licensed under the Creative Commons Attribution-NonCommercial-4.0 International License (CC BY-NC) (http://www.karger.com/Services/OpenAccessLicense), applicable to the online version of the article only. Usage and distribution for commercial purposes requires written permission.
College, London, using Fleiss' kappa statistic. The experts' teaching points were combined with operative footage and illustrative animations, and projected onto a virtual reality headset. The module was piloted to surgical science students ( $N=$ 15). Quantitative analysis compared participants' confidence regarding their anatomical knowledge before and after taking the module. Qualitative data were gathered from students regarding their views on using the virtual reality model. Results: Multi-rater agreement between experts was above the $70.0 \%$ threshold for all steps of the procedure. Seventy-three percentage of pilot study participants "agreed" or "strongly agreed" that they achieved a better understanding of surgical anatomy and the rationale behind each procedural step. This was reflected in an increase in the median knowledge score after trialing the curriculum $(p<0.001) .100 \%$ of subject-matter experts and $93.3 \%$ of participants "agreed" or "strongly agreed" that virtual mentorship would be useful for future surgical training. Conclusions: This study demonstrated that virtual surgical mentorship could be a feasible and cost-effective alternative to traditional training methods with the potential to improve technical skills, such as operative proficiency and nontechnical skills such as decision-making and situational judgement.

(c) 2021 The Author(s).

Published by S. Karger AG, Basel

Correspondence to:

Abdullatif Aydın, abdullatif.aydin@ @cl.ac.uk 


\section{Introduction}

The time-pressures of modern-day service commitments and the disruption to services caused by the COVID-19 pandemic mean that trainees spend less time in the operating theatre than ever before, making the traditional logbook-based approach of surgical training increasingly less feasible. Furthermore, increased levels of health literacy among patients and their families means that modern society expects surgical trainees to demonstrate an advanced level of competence, such as critical decision-making, at an earlier stage in their career.

Combining cutting-edge technologies such as virtual reality and evidence-based pedagogic approaches such as in-depth task analysis can help to develop new training methods that build upon time-honored surgical principles, such as surgical mentorship [1-4]. Cognitive task analysis is a technique used for instructional tool development, originally pioneered for high-stakes fields, such as aviation [5]. This streamlines the time-honored mentorship experience by providing a validated and structured framework for imparting precise and hermeneutic detail about expert performance standards [6].

Head-mounted display headsets project a virtual component at the user's eye-level, while allowing them to view and interact with their surroundings. Their ergonomic value has been demonstrated in the operating room, permitting surgeons to view patient scans intra-operatively, without having to shift their gaze from the patient to the monitor $[7,8]$. Beyond this, virtual reality headsets are portable high-fidelity simulators, providing exciting avenues for remote task training [9].

Our study aims to define key surgical anatomy and associated critical decision-making points for each stage of the selected surgical procedure. This was achieved by developing a virtual module by combining the experts' teaching points with visual graphics (e.g., operative footage and original 3D animation). Finally, the module underwent expert validation and piloting.

\section{Materials and Methods}

\section{Topic Selection}

The index procedure selected for this study was robot-assisted radical prostatectomy (RARP). This procedure accounts for a large proportion of the caseload in urology and has a high degree of anatomical and technical complexity [10]. This is further complicated by the presence of significant anatomical variation and the need to safely navigate through the limited field of view conferred by the minimally invasive approach [10].

HoloMentor: Virtual Reality for Remote Surgical Training

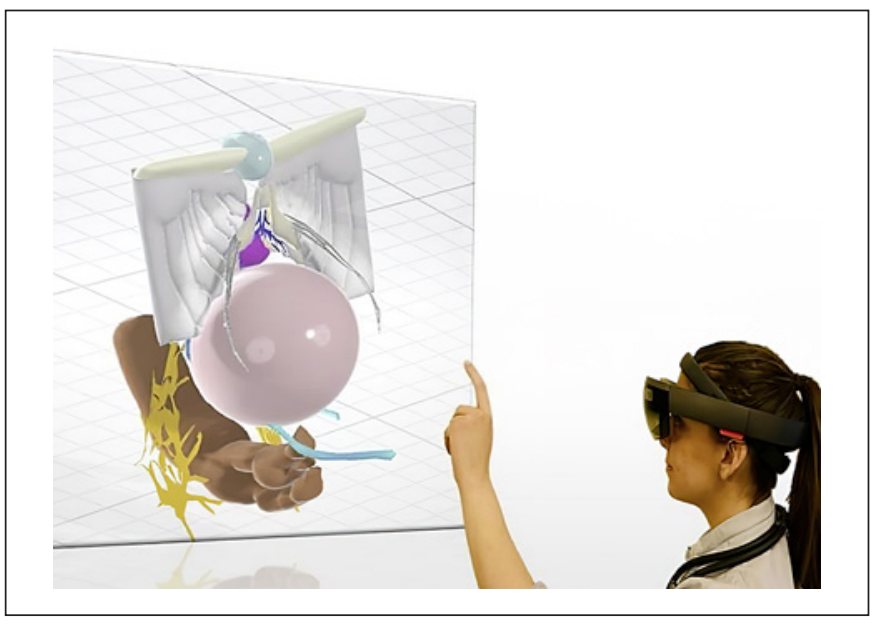

Fig. 1. Self-guided augmented reality module as seen through the HoloLens headset.

Virtual Mentorship Module Development

The Healthcare Failure Modes and Effect Analysis checklist for RARP is a validated procedure-specific guideline that outlines all the essential operative steps for the procedure [11]. Subject-matter experts $(N=3)$ at King's College London participated in semistructured interviews which lasted approximately $60 \mathrm{~min}$. For each step of the procedure, the experts discussed the following:

1. Goals and anatomical considerations.

2. Common hazards and errors.

3. Tips and techniques for error rescue and prevention.

These data were formatted into flowcharts, displaying the links between cognitive and practical performance standards.

Anonymized procedure-specific operative footage was obtained from the urology department at King's College London, and each step in the procedure was broken down into a "storyboard" of steps and matched with the appropriate expert commentary. Paint 3D (Microsoft, Redmond, WA, USA) and Blender Animation (Blender Foundation, Amsterdam, The Netherlands) were used to produce additional original $3 \mathrm{D}$ animations. This also allowed for depiction of any adverse scenarios, complications, or cues that were identified by the experts but were not demonstrated in the videos (shown in Fig. 1).

The experts' teaching points were converted into audio commentary using Adobe Voices (Adobe, San Jose, CA, USA) from the e-learning authoring software Adobe Captivate 9 (Adobe). During the module, this was transmitted through the virtual reality headset speakers as the learner viewed simulating the traditional intraoperative mentoring experience.

\section{Content Validity}

An independent panel of subject-matter experts at Imperial College, London $(N=8)$, was issued with an electronic, online survey and asked to rank the commentary for each operative step on a 1-5 Likert scale ( 1 being "least" and 5 being "most") for "Accuracy" and "Educational Utility." Fleiss' Kappa statistic for multirater agreement was computed. A percentage agreement, kappa value and 95\% CI were calculated on SPSS and GraphPad Prism 
8.0 (GraphPad Software Inc, San Diego, CA, USA). Any item scoring $<70 \%$ percentage agreement was revised.

\section{Pilot Study}

The pilot study sample comprised surgical science students. This group was selected for their ability to provide valuable feedback about the clarity of the module and its effectiveness as a virtual mentorship tool.

In March 2020, the pilot study took place at the Olympus Simulation Lab at Guy's Hospital during the Surgical Technology Symposium. Participants were recruited on a voluntary basis with no predetermined sample size.

In total, the module was attempted by 19 participants and fully completed by 15 participants, due to time constraints. The 4 remaining participants were excluded from the analysis. To minimize bias, all participants viewed the module and assessment questions for the first time during the pilot study. They were also asked to navigate through the module independently, thereby assessing the ease with which the virtual reality headset (Microsoft HoloLens) could be operated in the absence of an instructor. Before commencing the module, all participants had access to the brief video-instructional tutorial that is built-in to the headset teaching them the basic actions for operating the headset.

\section{Quantitative Analysis}

The module concluded with an assessment testing participants' understanding of the critical decision-making points and surgical anatomy. A minimum pass-mark of $80 \%$ was set for successful completion of the module.

Subsequently, participants were asked to complete an evaluative survey on their knowledge and confidence before and after the module. Participant scores were analyzed by a Wilcoxon signedrank test for confidence in anatomy and understanding of the procedural steps of the RARP procedure before and after the session.

\section{Qualitative Analysis}

Participants were also asked to rate the utility of virtual mentorship in surgical training based on their experience. The participants were also asked to provide qualitative feedback regarding the benefits and drawbacks of using virtual reality. Data were anonymized to reduce bias.

\section{Results}

\section{Content Validity}

Five of the 8 subject-matter experts completed validation (62.5\% response rate) rated the module for "Accuracy" $(k=0.96,95 \% \mathrm{CI}=0.87-1.00)$ and "Usefulness" $(k$ $=1,95 \% \mathrm{CI}=1.00-1.00) .100 \%$ of subject-matter experts "strongly agreed" that augmented reality will be a useful tool for surgical training.

\section{Pilot Study}

The pilot study recruited surgical science students ( $n$ $=15)$. The mean age of the students was $23.5( \pm 3)$ years.

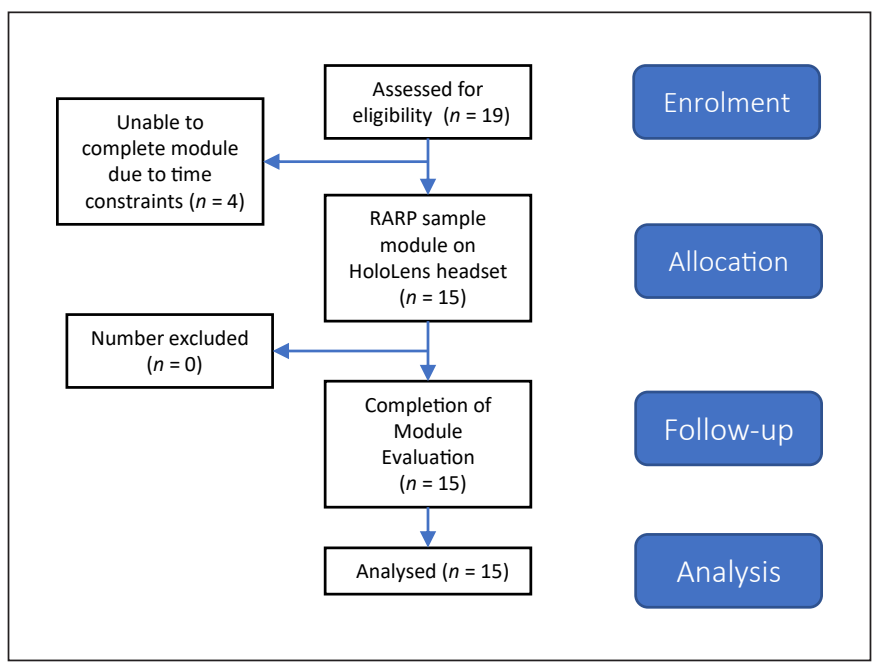

Fig. 2. Volunteer selection process flowchart.

Eight of the students were female and 7 were male. Study participation was voluntary (shown in Fig. 2).

\section{Module Evaluation: Quantitative Analysis}

Prior to trialing the module, the mean self-reported knowledge confidence score of participants regarding the regional surgical anatomy and understanding of the operation was 2.7 (on a scale from 1 to 5 , where $1=$ no knowledge and 5 = advanced knowledge), with $73 \%$ of participants reporting a score of 3 or less. After completing the module, the mean score increased to 3.9, with $100 \%$ of participants scoring $3-5$, indicating an increase by 1.2 points $(p<0.001)$ (shown in Fig. 3a).

Qualitative evaluation of the module revealed $93.4 \%$ of participants "strongly agreed" or "agreed" that their knowledge of the surgical anatomy had improved after the session. $86.7 \%$ of participants "strongly agreed" or "agreed" that the module improved their understanding of the operative steps and critical decision-making points (shown in Fig. 3b).

\section{Virtual Reality Headset Evaluation: Qualitative Analysis}

$80 \%$ of participants had no previous experience with virtual reality, HoloLens, or other head-mounted displays. The remaining $20 \%$ of participants had used other headsets, such as Oculus Rift and Google Glass but not HoloLens. 93.3\% of participants "strongly agreed" or "agreed" that virtual mentorship will be a useful addition to surgical training (shown in Fig. 3c).
Papalois et al. 


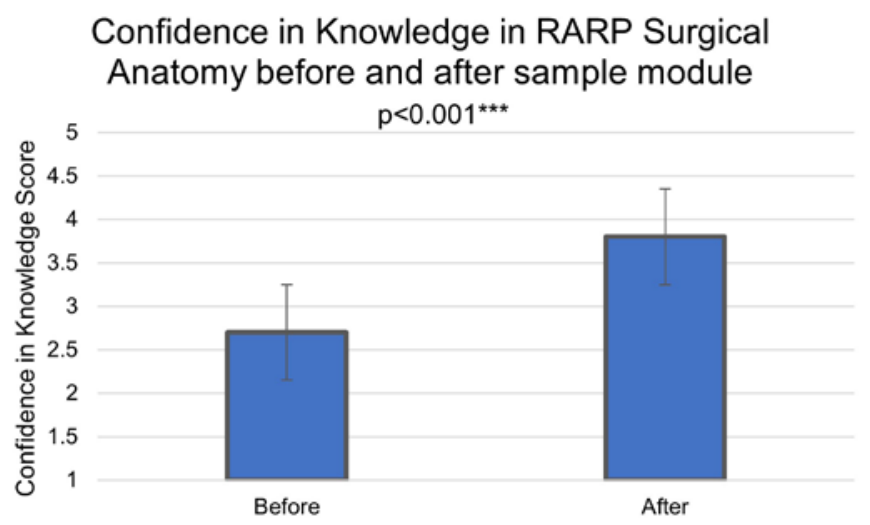

b 'My understanding of the rationale behind the steps of RARP has improved'

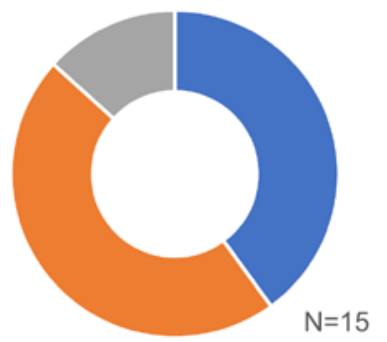

- Strongly Agree (40\%) - Agree (46.7\%) - Neutral(13.3\%) $=$ Disagree (0\%) $=$ Strongly Disagree $(0 \%)$

c 'Mixed reality will be a useful tool for surgical training'

Fig. 3. a Pre-post test confidence in knowledge of RARP surgical anatomy. b Understanding of RARP operative step sequence and rationale. c Perceived value of mixed reality in surgical training. RARP, robotassisted radical prostatectomy.

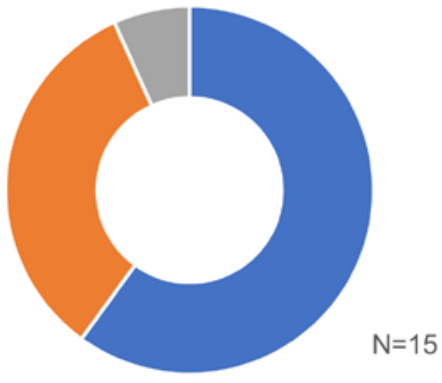

- Strongly Agree(60\%) $=$ Agree(33.3\%) $=$ Neutral(6.7\%) $\quad$ Disagree $(0 \%) \quad$ - Strongly Disagree $(0 \%)$
Participant feedback on the positive aspects of the augmented reality experience highlighted the usefulness of the holograms/3D animations and operative footage in understanding camera views and spatial relationships. Participant criticisms centered on headset weightiness, which limited the length of time for which it could be worn and the desire for more interactive elements and supplementation of the holograms with operative footage.

HoloMentor: Virtual Reality for Remote Surgical Training

\section{Discussion/Conclusion}

The 2020 COVID-19 pandemic has highlighted the need for flexible learning modalities that enable clinicians to acquire new competencies remotely, rapidly and reliably [12]. The findings of this study align with the results of previous studies on the benefits and criticisms of virtual reality and task analysis in surgical training and medical education $[13,14]$. 
Task analysis and detailed analysis of the practical framework for critical decision-making cultivated by experts over years' worth of experience provide a "remote proctoring" experience, extending the role of virtual reality beyond that of simple task-trainers, as most commonly reported in the literature, to tools for cultivating advanced nontechnical competencies, such as situational judgement [14]. Subsequently, an advanced level of surgical competence becomes attainable at a much earlier stage.

Furthermore, the virtual environment provides an ideal platform and safe space for explorative learning within complex scenarios [15]. In addition, the ability to record trainee performance and assessment scores using the inbuilt headset camera allows for thorough educational review and reflection between trainees and their clinical supervisors.

Owing to the good reusability factor of virtual reality headsets one or 2 headsets per teaching hospital are sufficient to provide access to several trainees [16]. Furthermore, the self-directed delivery of the modules and intuitive nature of the headsets minimize the trainer: trainee ratio, compared to traditional teaching methods and contribute to the cost-effectiveness of this investment. Nevertheless, the virtual mentoring module can also be easily adapted for more readily available interfaces, such as mobile phones, tablets, and computers, as exemplified by the commercially available Touch Surgery ${ }^{\mathrm{TM}}$ (Digital Surgery, London, UK) [17] smart-phone application which combines stepwise cognitive-task analysis style guidance with $3 \mathrm{D}$ illustrations for mobile phones and tablets. Furthermore, by providing a standardized remoteproctoring framework, there is potential to "democratize" access to surgical training opportunities by sharing such modules with institutions around the world, for example, virtually connecting surgeons in specialist centres with smaller centres, sharing knowledge, reducing variability in quality, and improving access to specialist services $[18,19]$.

Building upon the findings of the pilot study, further steps in the form of a randomized controlled trial, featuring a larger sample and a standard control group, for example, viewing the same content on a monitor or other non-head-mounted display, may be beneficial. Furthermore, to prevent potential reporting bias between the participants' self-reported confidence in knowledge and their actual level of knowledge, it may be beneficial to implement a pre-/post-intervention assessment with standardized correct answers. In addition, while pilot study participants in this study noted interactivity as a strength of the curriculum, there was a desire to expand and develop this further. In response, this prototype will be further refined for this parameter and adapted for the new Microsoft HoloLens 2 model.

In conclusion, a statistically significant increase in confidence and knowledge of the surgical anatomy and critical decision-making related to the surgical procedure was reported by the pilot study participants. Moreover, there was nearly unanimous consensus among the study participants and subject-matter experts that demonstrates significant learner/trainer satisfaction and suggests that virtual surgical mentorship and virtual reality headsets will be valuable tools in the future of surgical education.

Proposals for refining the process and product of surgical training must consider both the curriculum content and the interface of delivery. This requires high-fidelity curricula with evidence-based educational impact. These must be developed in a systematic and reproducible manner, minimizing costs and optimizing efficiency of delivery. The paradigm shift in surgical training from "modern apprenticeship" to "point of need learning" reflects a necessity to fulfil novel educational needs, while retaining time-honoured principles in surgical education, such as mentorship.

\section{Acknowledgements}

We are most grateful for the hardwork, support, and guidance of the research collaborators who participated in the data collection process and enabled this research to take place.

\section{Statement of Ethics}

Ethics approval was not required as data collection involved nonsensitive, completely anonymous educational tests, survey, and interview procedures. Participation was entirely voluntary, and verbal informed consent was obtained from all research collaborators.

\section{Conflict of Interest Statement}

The authors have no conflicts of interest to declare.

\section{Funding Sources}

This research was funded by the Royal College of Surgeons.
Papalois et al. 


\section{Author Contributions}

Zoe-Athena Papalois contributed to conceptualization, data Curation, formal analysis, investigation, methodology, writing (original draft), writing (review and editing);Abdullatif Aydın contributed to conceptualization, data curation, formal analysis, investigation, methodology, project supervision, writing (review and editing); Azhar Khan, FRCS (Urol), contributed to data curation, resources, and validation;Evangelos Mazaris, $\mathrm{MD}, \mathrm{PhD}, \mathrm{MSc}$ (Urol), FEBU, contributed to data curation, resources, and validation; Anand Muthusamy, FRCS, contributed to data curation, resources, and validation; Frank J.M.F., Dor MD, PhD, FRCS, FEBS (hon), contributed to data curation, resources, validation, and writing (review and editing); Prokar Dasgupta, MSc (Urol), MD, DLS, FRCS (Urol), FEBU, contributed to project supervision, data curation, resources, and validation; Kamran Ahmed, PhD, FRCS (Urol), contributed to project supervision, data curation, resources, validation, and writing (review and editing).

\section{Data Availability Statement}

Data available on request from the authors. Full CTA flowcharts are available on request.

\section{References}

1 Robinson R, O’Flynn KJ. Indicative operative numbers in urology training in the UK and Ireland. J Clin Urol. 2015;8(3):188-95.

2 Speroni KG, Fisher J, Dennis M, Daniel M. What causes near-misses and how are they mitigated? Plast Surg Nurs. 2014;34(3):114-9.

3 Giuse NB, Koonce TY, Storrow AB, Kusnoor SV, Ye F. Using health literacy and learning style preferences to optimize the delivery of health information. J Health Commun. 2012; 17(Suppl 3):122-40.

4 Aydin A, Fisher R, Khan MS, Dasgupta P, Ahmed K. Training, assessment and accreditation in surgery. Postgrad Med J. 2017; 93(1102):441-8.

5 Wingfield LR, Kulendran M, Chow A, Nehme J, Purkayastha S. Cognitive task analysis: bringing olympic athlete style training to surgical education. Surg Innov. 2014;22(4):40617.

6 Tofel-Grehl C, Feldon DF. Cognitive task analysis-based training. J Cogn Eng Decis Mak. 2013;7(3):293-304

7 Mentler T, Wolters C, Herczeg M. Use cases and usability challenges for head-mounted displays in healthcare. Curr Dir Biomed Eng. 2015;1(1):534-7.
8 Sanderson PM, Watson MO, Russell WJ, Jenkins S, Liu D, Green N, et al. Advanced auditory displays and head-mounted displays: advantages and disadvantages for monitoring by the distracted anesthesiologist. Anesth Analg. 2008;106(6):1787-97.

9 Stefanidis D, Sevdalis N, Paige J, Zevin B, Aggarwal R, Grantcharov T, et al. Simulation in surgery: what's needed next? Ann Surg. 2015; 261(5):846-53.

10 Pertuz Genes W, Claudia bicudo M, Pablo Miranda Machado P, Rios Auad P, Echavez Pacheco L, de Campos Zampolli $\mathrm{H}$, et al. Retzius Sparing (RS) Robotic Assisted Radical Prostatectomy (RARP) and retzius space reconstruction technique after RARP improve urinary Continence Compared to Conventional RARP (CRARP): systematic review and metanalysis. Int Arch Urol Complicat. 2019; $5(2)$.

11 Lovegrove C, Novara G, Mottrie A, Guru KA, Brown M, Challacombe B, et al. Structured and modular training pathway for Robot-assisted Radical Prostatectomy (RARP): validation of the RARP assessment score and learning curve assessment. Eur Urol. 2016;69(3): 526-35.

12 Daodu O, Panda N, Lopushinsky S, Varghese TK, Brindle M. COVID-19: considerations and implications for surgical learners. Ann Surg. 2020;272(1):e22-3.
13 Al Janabi HF, Aydin A, Palaneer S, Macchione N, Al-Jabir A, Khan MS, et al. Utilisation of the HoloLens mixed-reality device in minimally invasive surgery. Eur Urol Suppl. 2019; 18(1):e676-7.

14 Mattes-O’Brien C, Menozzi M. Practical application of hierarchical task analysis and cognitive work analysis in a surgical training system design. Zeitschrift für Arbeitswissenschaft. 2019;73(3):337-49.

15 Rae AO, Khatib M, Sarker S, Bello F. Cognitive task analysis performance of surgical trainees using an open hernia repair simulator. Int J Surg. 2015;23:S16-7.

16 Mushtaq J. Through the looking glass: a systematic review of intraoperative head mounted displays. Int J Surg. 2015;23:S70.

17 Sugand K, Mawkin M, Gupte C. Validating Touch Surgery ${ }^{\mathrm{mm}}$ : a cognitive task simulation and rehearsal app for intramedullary femoral nailing. Injury. 2015;46(11):2212-6.

18 Patil S, Chamberlain R. The financial burden of training surgical residents: who should bear the cost? J Surg Res. 2012;172(2):292.

19 Forster JA, Browning AJ, Paul AB, Biyani CS Surgical simulators in urological training: views of UK training programme directors. BJU Int. 2012;110(6):776-8. 